VOL. $13(1975), 429-437$.

\title{
On a system of Feferman
}

\section{Luiz Paulo de Alcantara}

A system of set theory which appears as an extension of Ackermann set theory is introduced. In this sytem we construct a syntactic model for a theory proposed by Feferman for the development of category theory.

Feferman introduced in [1] a system of axiomatic set theory. In this paper we consider a theory $T$, which can be viewed as an extension of Ackermann set theory (see, for example, [4]). Our main result is the construction in $T$ of a syntactic model for the system of Feferman. We shall use freely the terminology and notation of Feferman [1].

\section{Feferman's system}

The set theory of Feferman, $\mathrm{ZF} / \underline{s}$, is formulated in a first order language, $L_{\underline{s}}$, obtained by adding to. $L$ a constant symbol $\underline{s}$.

The axioms are taken to consist of the following (in the basic symbolism of $L_{s}$ )

(1) the axioms of $\mathrm{ZF}$,

(2) $\exists x(x \in \underline{s})$,

(3) $\forall x, y(y \in x \wedge x \in \underline{s} \rightarrow y \in \underline{s})$,

(4) $\forall x, y(x \in \underline{s} \wedge \forall z(z \in y \rightarrow z \in x) \rightarrow y \in \underline{s})$,

(5) $\forall x \in \underline{s}(\varphi(\underline{s})(x) \leftrightarrow \varphi(x))$, for each formula $\varphi$ of $L$ with free variables $x$.

Received 9 September 1975. The author is indebted to Professor T. Lucas of Louvain-la-Neuve for several comments and suggestions. 
(5) is a reflexion principle. What it means is the following:

suppose $(M, E, s)$ is a model of $\mathrm{ZF} / \underline{s}$; define

$M_{s}=\{x \mid x \in M$ and $x E s\}$, and say $x E_{s} y$ if and only if $x, y \in M_{s}$ and $x E y$. Then $(M, E)$ is an elementary extension of $\left(M_{s}, E_{s}\right)$.

\section{The system $T$}

The language $L_{u}$ of $T$ is obtained by adding to $L$ a constant symbol $u$.

The axioms are the universal closures of

(TI) $(\forall t)(t \in x \leftrightarrow t \in y) \rightarrow x=y$,

(т2) $(\exists t)(t \in u)$,

(T3) $(\forall x)(\exists y)(\forall t)(t \in y \leftrightarrow t \in x \wedge \varphi)$ where $\varphi$ is a formula of $L_{u}$ with free variables among $t, x$,

(T4) $y \in x \wedge x \in u \rightarrow y \in u$,

(T5) $x \in u \wedge(\forall t)(t \in y \rightarrow t \in x) \rightarrow y \in u$,

$(\forall x)(x \in u \wedge(\exists x)(\forall t)(\varphi(x, t)) \rightarrow t \in x) \rightarrow$

$$
(\exists z)(z \in u \wedge(\forall t)(\varphi(x, t) \rightarrow t \in z)) \text {, }
$$

for each formula $\varphi$ of $L$ with free variables $t, x$.

Let $\operatorname{Rn}(x, y)$ be the formula

$\operatorname{Ord}(x) \wedge(\exists z)((\forall w)(w \in x \rightarrow(\exists v) R l(w, v, z))$

$(\forall w, v)\left((w \in x \wedge \operatorname{RI}(w, v, x)) \vee(w=x \wedge v=y) \rightarrow(\forall t)\left(t \in v \leftrightarrow\left(\exists w^{\prime}, v^{\prime}\right)\right.\right.$ $\left.\left.\left.\left(w^{\prime} \in w \wedge \operatorname{RI}\left(w^{\prime}, v^{\prime}, z\right) \wedge t \subset v^{\prime}\right)\right)\right)\right)$,

where $\mathrm{Rl}(x, y, z)$ is the formula

$$
(\exists v)(v=(x, y) \wedge v \in z) .
$$

(T7) $(\forall x)(\exists \beta)(\exists y)(\operatorname{Rn}(\beta, y) \wedge x \in y)$.

If we interpret $u$ as the class of all sets of Ackermann, we can easily show that in $T$ the axioms of $A$ hold (for the axioms of $A$ see Reinhardt [4]). 
DEFINITION 1. Let $\varphi$ be a sentence of $A$. The $u$-transform of $\varphi$ in $\mathrm{T}, \varphi_{u}$, is obtained by replacing in $\varphi$ every part of the form $x \in M$ by $x \in u$.

THEOREM 1. If $\varphi$ is a theorem of A then $\varphi_{u}$ is a theorem of T.

Proof. It is enough to show that the $u$-transforms of the axioms of $A$ are theorems in $\mathrm{T}$.

Trivially we have (Al) $u,(\mathrm{~A} 2)_{u}$, and $(\mathrm{A3})_{u}$.

The u-transform of (A4) is

$(\forall x \in u)((\forall t)(\varphi(t) \rightarrow t \in u) \rightarrow(\exists z)(z \in u \wedge(\forall t)(t \in z \leftrightarrow \varphi(t))))$.

Taking $x=u$, by (T6) we have

$$
(\exists z)(z \in u \wedge(\forall t)(\varphi(t) \rightarrow t \in z)) \text {. }
$$

Let $w=\{t \mid t \in u \wedge \varphi(t)\} . w \subset z$ and $z \in u$, so $w \in u$ and $(\forall t)(\varphi(t) \leftrightarrow t \in w)$.

As for Ackermann set theory, $u$ cannot be defined in $T$, using the language $L$, that is, if $\varphi(t, x)$ is a formula of $L$ we can show that

$$
\sim(\exists x)(x \in u \wedge(\forall t)(t \in u \leftrightarrow \varphi(t, x))) \text {. }
$$

In fact, suppose

$$
(\forall t)(t \in u \leftrightarrow \varphi(t, x)) \text { and } x \in u \text {. }
$$

By $(T 6)$,

$$
(\exists z)(z \in u \wedge \forall t(t \in z \leftrightarrow \varphi(t, x))) \text {. }
$$

By (TI), $z=u$ and then $u \in u$. By (T3),

$$
(\exists y)(\forall t)(t \in y \leftrightarrow t \in u \wedge t \vDash t),
$$

and we have $y \subset u$. Then $y \in u$ and we obtain a contradiction, $y \in y \leftrightarrow y \leqslant y$.

DEFINITION 2. Let $M=(M, U, R)$ be a model of $T$. We say that $A \subset M$ is definable in $(M, B)$ if and only if there is a formula $\varphi(v, x)$ of $L$ and elements $b$ of $B$ such that for every $t \in M$, $t \in A \leftrightarrow M F \varphi(t, b)$; that is,

$$
A=\{t \in M \mid M \models \varphi(t, \mathbf{b})\} \text {. }
$$


THEOREM 2. If $M=(M, U, R)$ is a model of $\mathrm{T}$, then $U$ is not definable in $(M, U)$.

Proof. Suppose $U$ is definable. Then there exists a formula $\varphi(v, x)$ of $L$ and elements $b$ of $U$ such that for every $t \in M$,

$$
t \in U \leftrightarrow M \vDash \varphi(t, \mathrm{~b}),
$$

that is $U=\{t \in M \mid M \models \varphi(t, b)\}$. Hence,

$$
M \models b \in u \wedge(\forall t)(\varphi(t, b) \rightarrow t \in u) \text {. }
$$

Since $M \models(T 6)$,

$$
M=\exists z(z \in u \wedge \forall t(t \in z \leftrightarrow \varphi(t, b))) .
$$

Hence there is $y \in U$ such that for every $t \in M$,

$$
t \in y \leftrightarrow M=\varphi(t, \text { b }) \text {. }
$$

Since $M F(T 1)$, by $(6)$ we have $y=U$ and $U \in U$.

We construct now in $\mathrm{T}$ a syntactic model of $\mathrm{ZF} / \underline{s}$.

DEFINITION 3. Let $\varphi$ be a sentence of $\mathrm{ZF} / \underline{s}$. The $u$-transform of $\varphi$ in $\mathrm{T}, \varphi_{\mathcal{U}}$, is obtained replacing in $\varphi$ every part of the form $x \in \underline{s}$ by $x \in u$.

THEOREM 3. If $\varphi$ is a theorem of $\mathrm{ZF} / \underline{s}$, then $\varphi_{u}$ is a theorem of T.

We need a lemma.

\section{LEMMA 1.}

(a) $\operatorname{Rn}(\alpha, y) \wedge \operatorname{Rn}\left(\alpha, y^{\prime}\right) \rightarrow y=y^{\prime} ;$

(b) $\mathrm{Rn}(\alpha, y) \rightarrow \mathrm{Sc}(y)$;

(c) $\alpha \in u \wedge y \in u \rightarrow(R n(\alpha, y) \leftrightarrow y=R(\alpha))$;

(d) $(\forall \alpha \in u)(\exists y \in u) \operatorname{Rn}(\alpha, y)$;

(e) $(\forall \alpha)(\exists y) \operatorname{Rn}(\alpha, y)$.

All (a)-(e) can be proved in $A$ (see Lévy [2] and Lévy and Vaught [3]), so, by Theorem 1 , also in $T$.

Proof of Theorem 3. It is enough to show in $T$ the $u$-transforms of the axioms of $\mathrm{ZF} / \underline{\underline{s}}$. 
Trivially we have (2) $u,(3)_{u}$, and (4) $u$.

The $u$-transform of (5) is

$$
(\forall x)(x \in u \rightarrow(\varphi(u)(x)) \leftrightarrow \varphi(x))) .
$$

If $\varphi$ has no quantifiers then $\varphi^{(u)}$ is simply $\varphi$.

Assume $\varphi$ of the form $\exists t \psi$, where $\psi$ is a formula of $L$ with free variables $t, x$. So we have to show that

$$
(\forall x)(x \in u \wedge(\exists t) \psi \rightarrow(\exists t \in u) \psi) .
$$

Assume the hypothesis. By (TT) there exists $\beta$ and $z$ such that $\operatorname{Rn}(\beta, z), \quad t \in z$ and $\psi$. Also by (T7) there exists $\alpha$ and $y$ such that $\operatorname{Rn}(\alpha, y)$ and $z \in y$. Let $a=\{x \mid \operatorname{Rn}(\alpha, y) \wedge x \in y \wedge \Phi(x)\}$, where $\Phi(x)$ is the formula $(\exists \gamma)(\operatorname{Rn}(\gamma, x) \wedge(\exists t \in x) \psi)$.

Since $z \in y, \operatorname{Rn}(\beta, z)$, and $\Phi(z), a$ is not empty. Therefore, there exists $b \in a$ such that

$$
\text { (i) }(\forall x)(x \in a \rightarrow x \notin b) \text {. }
$$

For $b$ we can prove

$$
\text { (ii) }(\forall x)(x \in a \rightarrow b \subset x) \text {. }
$$

In fact, since $b \in a, b \subset x$ or $x \subset b$. But if $x \subset b$, we have $x \in b$ because $\Phi(x)$, which contradicts (i). b is the set of elements $v$ such that for all $x, \Phi(x)$ implies $v \in x$; that is,

(iii) $\forall v(v \in b \leftrightarrow \forall x(\Phi(x) \rightarrow v \in x))$. In fact, if $\forall x(\Phi(x) \rightarrow v \in x)$, then $v \in b$, since $b \in a$, and then $\Phi(b)$.

Conversely, suppose $v \in b$ and $\Phi(x)$. We have $x \subset z$ or $z \subset x$. If $x \subset z \in y$ then $x \in y$ and $x \in a$. By (i), $b \subset x$ and then $v \in x$. If $z \subset x$, since $z \in a$, by (ii), $b \subset z \subset x$ and then $v \in x$.

Finally we apply (T6) to the formula

$$
(\forall x)(\Phi(x) \rightarrow t \in x) .
$$

By (iii), $t \in b$, and then

$$
(\exists z)(z \in u \wedge(\forall t)((\forall x)(\Phi(x) \rightarrow t \in x) \rightarrow t \in z)) .
$$


By $(T 4), b \subset z \subset u$ and $t \in u$.

It remains to show that the axioms of $\mathrm{ZF}$ are provable in $T$. It is enough to show their relativizations.

The extensionality axiom follows from (TI).

The empty set axiom follows from (T2) and (T5).

The unordered pairs axiom: let $\varphi$ be the formula $t=a \vee t=b . \varphi$ implies $t \in u$. By (T3) there exists $y$ such that $y=\{t \mid \varphi\}$. By (T6) there exists $z \in u$ such that $y \subset z$. Therefore, by (T5), $y=\{a, b\} \in u$.

The union set axiom: let $\varphi$ be the formula $t \in a \wedge a \in b$. We have $\varphi \rightarrow t \in u$ and there exists $y$ such that $y=\{t \mid \varphi\}$. By (T6) there exists $z \in u$ such that $y \subset z$. By (T5), $y=U b \in u$.

The power set axiom: let $\varphi$ be the formula $t \subset a . \varphi$ implies $t \in u$ and there exists $y$ such that $y=\{t \mid \varphi\}$. Therefore there exists $z \in u$ such that $y \subset z$. So we have $y=P a \in u$.

The axiom of infinity: let $\varphi(z)$ be the formula

$$
(\forall y)(0 \in y \wedge \forall t(t \in y \rightarrow t \cup\{t\} \in y) \rightarrow z \in y) \text {. }
$$

$\varphi(z)$ implies $z \in u$, since $0 \in u$ and $\forall t(t \in u \rightarrow t u\{t\} \in u)$. Then there exists $w \in u$ such that $w=\{z \mid \varphi(z)\}$. This $w$ can be easily seen to be as required by the axiom of infinity.

The replacement axiom schema: the relativization of the replacement axioms is as follows, for any formula $\varphi$ of $L$ with free variables $x, y, a, z$ in all:

$$
\begin{aligned}
\forall a, z \in u\left(\forall x, y_{1}, y_{2} \in\right. & \left.u\left(\varphi^{(u)}\left(x, y_{1}\right) \wedge \varphi^{(u)}\left(x, y_{2}\right) \rightarrow y_{1}=y_{2}\right)\right) \rightarrow \\
& (\exists b \in u)(\forall y \in u)\left(y \in b \leftrightarrow \exists x\left(x \in a \wedge \varphi^{(u)}(x, y)\right)\right) .
\end{aligned}
$$

Let $\psi$ be the formula $\left(\forall x, y_{1}, y_{2}\right)\left(\varphi\left(x, y_{1}\right) \wedge \varphi\left(x, y_{2}\right) \rightarrow y_{1}=y_{2}\right) \rightarrow$ $(\exists b)(\forall y)(y \in b \leftrightarrow \exists x(x \in a \wedge \varphi(x, y)))$.

By $(5)_{u}$ we have 
(i) $z, x, y \in u \rightarrow\left(\varphi \leftrightarrow \varphi^{(u)}\right)$, and

(ii) $z, x \in u \rightarrow\left(\exists y \varphi \leftrightarrow(\exists y \in u) \varphi^{(u)}\right)$.

Then,

(iii) $\quad z, x \in u \rightarrow(\exists y \varphi \leftrightarrow(\exists y \in u) \varphi)$.

Let $\varphi$ be a function and $b=\{y \mid \exists x \in a \varphi(x, y)\}$. By (iii), $b \in u$ since $a \subset u$ and then $x \in u$. Therefore

$$
z, a \in u \rightarrow \psi \text {. }
$$

But, by $(5)_{u}, z, a \in u \rightarrow\left(\psi \leftrightarrow \psi^{(u)}\right)$. Then $z, a \in u \rightarrow \psi^{(u)}$ and this completes the proof.

The foundation axiom follows from (T7) and Lemma 1. The proof is now complete.

Consider the theory $\mathrm{T}^{\prime}$ obtained from $\mathrm{T}$ by replacing (T3) by

(T3)' $(\forall x)(\forall x)(\exists y)(\forall t)(t \in y \leftrightarrow t \in x \wedge \varphi(t, x))$, for each formula $\varphi$ of $L$ with free variables $t, x$.

In $Z F / \underline{s}$ we can construct a syntactic model of $\mathrm{T}^{\prime}$.

DEFINITION 4 . Let $\varphi$ be a sentence of $T^{\prime}$. The $\underline{s}$-transform of $\varphi$ in $\mathrm{ZF} / \underline{s}, \varphi_{\underline{s}}$, is obtained replacing in $\varphi$ every part of the form $x \in u$ by $x \in \underline{s}$.

THEOREM 4. If $\varphi$ is a theorem of $\mathrm{T}^{\prime}$, then $\varphi_{\underline{s}}$ is a theorem of $\mathrm{ZF} / \underline{\underline{s}}$.

Proof. It is enough to show in $\mathrm{ZF} / \underline{s}$ the $\underline{s}$-transforms of the axioms of 'T'.

Evidently we have $(\mathrm{TI})_{\underline{s}},(\mathrm{~T} 2)_{\underline{s}},(\mathrm{~T} 3)_{\underline{\underline{s}}},(\mathrm{~T} 4)_{\underline{s}}$, and $(\mathrm{T} 5)_{\underline{s}}$. We show $(T 6)_{\underline{s}} ;$ that is

$(\forall x)((x \in s \wedge(\exists x)(\forall t)(\varphi(t, x)) \rightarrow t \in x)) \rightarrow$

$$
(\exists z)(z \in s \wedge(\forall t)(\varphi(t, x) \rightarrow t \in z)) \text {. }
$$

Assume the hypothesis. Let $\psi$ be the formula

$$
(\forall t)(\varphi(x, t) \rightarrow t \in x) .
$$

By $(5)$, 


$$
x \in \underline{s} \rightarrow((\exists x)(\forall t)(\varphi \rightarrow t \in x) \leftrightarrow(\exists x \in \underline{s})(\forall t \in \underline{s})(\varphi(\underline{s}) \rightarrow t \in x)) .
$$

Hence

$$
\text { (i) }(\exists x \in \underline{s})(\forall t \in \underline{s})(\varphi(\underline{s}) \rightarrow t \in x) \text {. }
$$

We also have

$$
\begin{aligned}
& \text { (ii) }(\forall t \in \underline{s})(\varphi(\underline{s}) \rightarrow t \in x) . \\
& \text { Since } x \in \underline{s} \text { and } x \in \underline{s}, \\
& \text { (iii) } \psi \leftrightarrow \psi^{(\underline{s})} \text {. }
\end{aligned}
$$

Then by (ii) we obtain $\psi$ and this completes the proof.

Finally we show $(T 7)_{\underline{s}}$.

Let $\sigma=\{\alpha \mid \alpha \in \underline{s} \wedge \operatorname{Ord}(\alpha)\}$. Then $\sigma$ is an ordinal and is the least one not in $\underline{s}$. Moreover, $\sigma$ is a limit ordinal with $\sigma>\omega$, and furthermore we have $\underline{s}=R(\sigma)$.

By $(5),(T 7)_{\underline{s}}$ is equivalent to

$$
(\mathrm{T} 7)_{\underline{s}}^{(\underline{s})} \forall x \in \underline{s} \exists y \in \underline{s} \exists z \in \underline{s}(\operatorname{Rn}(y, z) \wedge x \in z),
$$

and this follows from Lemma 1 and the fact that $\underline{s}=R(\sigma)$.

The proof is now complete.

\section{References}

[1] Solomon Feferman, "Set-theoretical foundations of category theory", Reports of the Midwest Category Seminar III, 201-232 (Lecture Notes in Mathematics, 106. Springer-Verlag, Berlin, Heidelberg, New York, 1969).

[2] Azriel Lévy, "On Ackermann's set theory", J. Symbolic Logic 24 (1959), $154-166$.

[3] A. Lévy and R. Vaught, "Principles of partial reflection in the set theories of Zermelo and Ackermann", Pacific J. Math. 11 (1961), 1045-1062. 
[4] William N. Reinhardt, "Ackermann's set theory equals ZF", Ann. Math. Logic 2 (1970), 189-249.

Institut de Mathématiques,

Université de Louvain,

Belgium;

Instituto de Matemática,

Universidade Estadual de Campinas,

Brasil. 